\title{
Ribozyme RPI.4610
}

National Cancer Institute

\section{Source}

National Cancer Institute. Ribozyme RPI.4610. NCI Thesaurus. Code C1865.

A nuclease-stabilized synthetic ribozyme (ribonucleic acid enzyme) with potential antiangiogenesis activity. Ribozyme RPI.4610 specifically recognizes the mRNA for FLT1 (vascular endothelial growth factor receptor 1; VEGFR1), and hydrolyzes the mRNA, thereby preventing VEGFR1 proteins from being made. This may prevent VEGFstimulated angiogenesis in cancerous tissue and metastasis. 